\title{
Concentração de lactato sérico em ovelhas e cordeiros mestiços (1/2 Suffolk) nascidos em eutocia
}

\author{
[Lactate blood levels in sheep and newborn lambs (1/2 suffolk) in normal delivery] \\ L.P. Silva ${ }^{1}$, M.L.G. Lourenço ${ }^{1 *}$, M.C. Grandi ${ }^{2}$, C.M. Vela Ulian ${ }^{1}$, \\ M.J. Sudano ${ }^{1}$, S.B. Chiacchio ${ }^{1}$ \\ ${ }^{1}$ Faculdade de Medicina Veterinária e Zootecnia - Universidade Estadual Paulista \\ Júlio de Mesquita Filho - Botucatu, SP \\ ${ }^{2}$ Fazenda Experimental Marcello Mesquita Serva - Universidade de Marília - Unimar - Marília, SP
}

\begin{abstract}
RESUMO
O objetivo do estudo foi avaliar a concentração de lactato sanguíneo venoso em ovelhas e em cordeiros neonatos imediatamente após o parto eutócico. Foram utilizadas 14 ovelhas, sendo nove da raça Suffolk e quatro mestiças e 20 cordeiros neonatos sadios (machos e fêmeas). $O$ efeito do sexo, da raça e do número de cordeiros por parto foi investigado, bem como a possível correlação entre a concentração de lactato materno e neonatal. Não houve diferença entre machos e fêmeas, em relação à raça (Suffolk e mestiços), contudo a concentração de lactato foi mais elevada em cordeiros gêmeos ou trigêmeos quando comparado a cordeiros uníparos. Houve correlação positiva entre a concentração materna e a neonatal, sendo que quanto maior a concentração de lactato materno, maior a concentração no cordeiro imediatamente após o parto eutócico. Os resultados do presente estudo fornecem um subsídio por meio do qual se podem comparar os níveis de lactato sanguíneo em cordeiros em estado crítico.
\end{abstract}

Palavras-chave: hiperlactatemia, ovinos, neonatos, parto eutócico

\begin{abstract}
The aim of this study was to evaluate the venous blood lactate concentration in sheep and newborn lambs immediately after normal delivery. We used 14 ewes, nine and four Suffolk crossbred lambs and 20 healthy newborns (males and females). The effect of gender, race and number of lambs per birth was investigated, as well as the possible correlation between the concentration of maternal and newborn lactate. There was no difference between males and females or in relation to race (and Suffolk crossbred); however the concentration of lactate was higher in twin or triplet lambs when compared to single lambs. There was a positive correlation between the maternal and newborn concentration and the higher the concentration of lactate, the greater the concentration in the lamb immediately after normal delivery. The results of this study provide a base from which to compare the blood lactate levels in lambs in critical condition.
\end{abstract}

Keywords: hyperlactatemia, sheep, newborn, eutocic partum

\section{INTRODUÇÃO}

A mortalidade perinatal em cordeiros representa um problema biológico e economicamente sério para a produção mundial de ovinos. Apesar da melhora acentuada nos aspectos nutricionais e no controle sanitário, uma das grandes preocupações na criação de ovinos no Brasil e no mundo é a elevada taxa de mortalidade em cordeiros nos primeiros dias de vida, diminuindo os ganhos produtivos (Rook et al., 1990; Cloete e Scholtz, 1998).

Recebido em 1 de março de 2012

Aceito em 6 de março de 2013

* Autor para correspondência (corresponding author)

E-mail: mege@uol.com.br 
Em rebanhos ovinos, bem como em outras espécies, o período neonatal exige grandes cuidados, pois as causas da elevada taxa de mortalidade ainda permanecem obscuras. Segundo Dutra et al. (2007), lesões no sistema nervoso central representam uma causa importante da perda perinatal, uma vez que cordeiros que vêm a óbito entre o nascimento e os cinco dias de idade apresentam lesões neurológicas resultantes de encefalopatia hipóxico-isquêmica de gravidade variável. Os índices de mortalidade durante o período perinatal apontam a asfixia perinatal como uma causa importante da mortalidade em cordeiros (Dutra e Banchero, 2011).

A asfixia neonatal, definida como condição em que há deficiência nas trocas gasosas sanguíneas, leva à hipoxemia progressiva e à hipercapnia com acidose metabólica significativa (Low, 1996), sendo uma causa comum de morbidade e mortalidade também em recém-nascidos humanos (Volpe, 2001; Koliski et al., 2007), suínos (Randall, 1971; Herpin et al., 1996), bovinos (Bleul et al., 2008) e equinos (Vaala, 1999). Nos partos prolongados, a asfixia afeta a capacidade dos cordeiros recém-nascidos em levantar-se e procurar o úbere o mais breve possível (entre 15 minutos e duas horas) para ingestão de colostro (Dwyer, 2008), bem como é associada ao aumento da mortalidade perinatal nos partos gemelares (Everett-Hincks et al., 2007; Dutra e Banchero, 2011).

Na obstetrícia humana, o lactato desempenha um papel central como marcador de sofrimento fetal e neonatal. A presença de lactato em níveis excessivos documenta a utilização de vias secundárias de oxigenação devido à hipóxia decorrente de eventos que ocorreram durante o parto (Saugstad, 2002; Groppetti et al., 2010). Sua determinação em recém-nascidos é utilizada nos casos de encefalopatia hipóxica-isquêmica, monitorização do tratamento com fluidos e agentes inotrópicos/vasopressores, e acompanhamento da progressão de doenças (Magdesian, 2003).

Sendo o lactato considerado, portanto, um bom indicador de hipóxia tecidual no período neonatal precoce, sua utilização tem se tornado cada vez mais importante, para a monitoração de pacientes em emergências, como choque hipovolêmico, séptico, inclusive no paciente anestesiado, como indicador de perfusão tecidual adequada (Floriano et al., 2010).

Estudos sobre a asfixia durante o nascimento em cordeiros recém-nascidos são escassos, provavelmente devido à impossibilidade de realização de análises laboratoriais e de lactato imediatamente após colheita de sangue, o que não é possível em fazendas localizadas longe de laboratórios clínicos especializados (Peiró et al., 2010; Dutra e Banchero, 2011). Com o uso de medidores portáteis economicamente acessíveis, a avaliação da lactatemia vem se tornando uma ferramenta extremamente útil em hospitais veterinários, para se determinar a gravidade de determinadas circunstâncias, bem como para guiar as decisões terapêuticas (Belettini et al., 2008).

O objetivo do presente estudo foi, portanto, descrever os níveis de lactato venoso em ovelhas e em seus respectivos cordeiros neonatos imediatamente após o parto eutócico.

\section{MATERIAL E MÉTODOS}

Foram examinadas amostras de sangue de 20 cordeiros neonatos imediatamente ao nascimento (11 fêmeas; nove machos), sendo 13 da raça Suffolk e sete sem raça definida, com peso médio de quatro quilos, e de 14 ovelhas, sendo nove da raça Suffolk e quatro sem raça definida, com peso médio de 50 a 60 quilos. A distribuição quanto ao número de cordeiros por ninhada foi: cinco gêmeos (10 cordeiros), e 10 neonatos uníparos. Os animais eram provenientes da cabanha da Fazenda Experimental Marcello Mesquita Serva da Universidade de Marília Unimar.

As ovelhas foram criadas a pasto em sistema de pastejo rotacionado, sendo todas devidamente vacinadas e vermifugadas. No final da gestação, as fêmeas foram identificadas e separadas próximo ao parto, sendo recolhidas do pasto com seus cordeiros após o nascimento. Os cordeiros e as ovelhas foram levados a um piquete menor fixo, onde se procederam à colheita de sangue (cinco a 15 minutos após o nascimento), à identificação, ao exame clínico, à pesagem e à antissepsia do umbigo. A inclusão dos animais utilizados no estudo foi aleatória, de acordo com o nascimento. 
A coleta de sangue, neonatal e materna, foi realizada mediante a punção da veia jugular, utilizando-se o sistema de coleta a vácuo (Vacutainer, Becton Dickinson, Brasil), em frascos de $5 \mathrm{~mL}$ contendo fluoreto de sódio/ácido etilenodiaminotetracético dissódico (NaF/Na2EDTA). As amostras foram mantidas sob refrigeração até a chegada no laboratório, quando foram centrifugadas a $1.000 \mathrm{x} \mathrm{g}$, durante cinco minutos, obtendo-se o plasma necessário para a análise laboratorial.

Os testes foram realizados utilizando-se reagente de uso comercial (Kit LOP-PAD, Katal, Brasil), e a leitura do parâmetro bioquímico foi realizada em espectrofotômetro semiautomático (Labquest, Labtest Diagnóstica, Brasil), em comprimentos de onda específicos para lactato.

Os dados foram analisados utilizando-se a ANOVA, por meio do PROC GLM do SAS (SAS Inst. Inc., Cary, NC, USA). As fontes de variação no modelo, incluindo sexo (fêmea e macho), raça (Suffolck e sem raça definida), número de cordeiros por ninhada (único, gêmeo, trigêmeo) e interações de primeira ordem, foram consideradas como efeitos fixos. Os dados foram apresentados como média dos quadrados mínimos e erro-padrão. $\mathrm{Na}$ ausência de interações significativas, apenas os efeitos principais foram apresentados. A correlação de Pearson foi calculada entre a concentração de lactato materna e dos cordeiros. Para todas as análises foi adotado o nível de significância de $5 \%$.

O estudo foi aprovado pela Comissão de Ética no Uso de Animais da FMVZ/Unesp/Campus de Botucatu (Protocolo n ${ }^{\circ}$ 152/2010).

\section{RESULTADOS E DISCUSSÃO}

Os valores de lactato sérico em cordeiros neonatos $(2,9 \pm 0,3 \mathrm{mmol} / \mathrm{L})$ e em ovelhas $(3,0 \pm 0,5 \mathrm{mmol} / \mathrm{L}), \operatorname{logo}$ após o parto eutócico, foram superiores aos limites de referência estabelecidos para espécie ovina, cuja variação, segundo Kaneko (1997), é de 1,00 a $1,33 \mathrm{mmol} / \mathrm{L}$. Os valores médios obtidos foram elevados e semelhantes aos descritos por outros autores em cordeiros. Em 1972, Comline e Silver avaliaram a concentração de lactato plasmático venoso em 10 ovelhas da raça Welsh e em seus respectivos cordeiros no período pré-natal e imediatamente após o parto, sendo que, ao nascimento, a concentração foi de 3,88 $4,4 \mathrm{mmol} / \mathrm{L}$ para os cordeiros e $2,88 \mathrm{mmol} / \mathrm{L}$ para as ovelhas; segundo os autores, os níveis de lactato materno durante o parto foram mais elevados do que nos dias que o antecederam, contudo inferiores quando comparados aos dos fetos.

Semelhante a outras espécies, como humanos (Volpe, 2001), suínos (Randall, 1971; Herpin et al., 1996), bovinos (Bleul et al., 2008) e equinos (Vaala, 1999), o presente estudo demonstrou que a concentração média de lactato em ovelhas e em cordeiros ao nascimento encontrava-se acima dos valores de referência. Essa elevação ao nascimento é fisiológica e deve-se à estimulação generalizada do sistema nervoso simpático com liberação de adrenalina e de cortisol, durante e após o nascimento, promovendo não somente o aumento de lactato como também do volume globular, da glicose, da frequência cardíaca e dos ácidos graxos livres, conforme proposto por Comline e Silver (1972). A hipóxia fisiológica induzida durante o processo do nascimento também proporciona a elevação do lactato.

Thorngren-Jerneck et al. (2001) estudaram o efeito da asfixia fetal da injúria hipóxicoisquêmica cerebral em cordeiros. A concentração média de lactato arterial nos cordeiros sob asfixia induzida no momento do parto $(12,7 \mathrm{mmol} / \mathrm{L})$, aos cinco $(14,9 \mathrm{mmol} / \mathrm{L})$ e aos 10 minutos $(15,6 \mathrm{mmol} / \mathrm{L})$ após o nascimento, foi mais elevada quando comparada ao grupo-controle sem asfixia avaliado aos cinco e aos 10 minutos $(12,1$ e $12,3 \mathrm{mmol} / \mathrm{L}$, respectivamente). Quatro horas após o parto, houve queda acentuada dos níveis de lactato nos cordeiros do grupo-controle $(7,9 \mathrm{mmol} / \mathrm{L})$ em relação aos neonatos submetidos à asfixia $(11,5 \mathrm{mmol} / \mathrm{L})$. A diminuição do lactato pode ser justificada pela depuração gradativa e pelo consumo do lactato por meio da conversão em piruvato para a produção de energia, indicando uma reversão da hipóxia tecidual a que o neonato foi submetido até o momento do parto e um melhor prognóstico (Souza e Elias, 2006).

Não houve efeito significativo da raça $(\mathrm{P}=0,8098)$ ou do sexo $(\mathrm{P}=0,5783)$ sobre a concentração de lactato, não existindo interação significativa entre o sexo e a raça $(\mathrm{P}=0,0845)$, entre o sexo $(\mathrm{P}=0,3575)$ ou a raça $(\mathrm{P}=0,8441)$ 
(Tab. 1). De acordo com Dutra e Banchero (2011), a probabilidade para o desenvolvimento de asfixia é influenciada pela duração do parto, pelo tamanho da ninhada e pela raça da ovelha. A duração do parto é menor no nascimento de fêmeas do que em machos e também em gêmeos em relação aos cordeiros únicos, havendo interação entre o sexo e o tamanho da ninhada. No presente estudo, é provável que a inexistência da interação entre lactato e sexo seja devido ao número de animais avaliados, pequeno para identificação de tal interação. Por se tratarem também de cordeiros mestiços (1/2 Suffolk), a diferença do peso ao nascimento, entre machos e fêmeas, talvez não tenha sido tão evidente quanto à descrita em raças puras como a Texel e a Polwart.

Tabela 1. Média, erro-padrão e efeito sobre a concentração de lactato em cordeiros neonatos de acordo com a raça (Suffolk e mestiços), o sexo (machos e fêmeas) e o número de filhotes (pluríparos ou uníparos)

\begin{tabular}{cccc}
\hline Raça filhote & Média lactato & Erro-padrão & Valor de P \\
Suffolck & 2,7 & 0,4 & 0,8098 \\
Mestiços & 3,5 & 0,5 & \\
\hline Sexo cordeiro & Média lactato & Erro-padrão & Valor de P \\
\hline Macho & 3,3 & 0,5 & 0,5783 \\
Fêmea & 2,8 & 0,5 & \\
\hline Raça filhote & Média lactato & Erro-padrão & 0,0289 \\
Trigêmeos & $3,9 \mathrm{a}$ & 0,4 & \\
Gêmeos & $2,7 \mathrm{ab}$ & 0,5 & \\
Filhote único & $1,7 \mathrm{~b}$ & 0,8 & \\
\hline
\end{tabular}

Letras sobrescritas distintas na mesma coluna diferem entre si ao nível de 5\% de probabilidade $(\mathrm{P}<0,05)$.

Cordeiros nascidos de ovelhas da raça Texel apresentam $9 \%$ de probabilidade de asfixia quando comparados aos da raça Polwarth. No estudo descrito por esses autores, embora o lactato não tenha sido avaliado, pela maior probabilidade de asfixia ao nascimento nestas raças, provavelmente haveria efeito da raça, da duração do parto e do tamanho da ninhada sobre os níveis de lactato.

Ocorreu efeito do número de cordeiros sobre a concentração de lactato $(\mathrm{P}=0,0289)$, sendo esta significativamente maior nos cordeiros gêmeos quando comparada aos cordeiros únicos (Tab. 1). Os resultados do presente estudo estão de acordo com Stafford et al. (2007), que analisaram a concentração de lactato em cordeiros da raça Romney ao nascimento de acordo com o tamanho da ninhada. A concentração média de lactato em nosso estudo em cordeiros trigêmeos $(2,51 \mathrm{mmol} / \mathrm{L})$ foi significativamente mais elevada quando comparada aos gêmeos $(2,15 \mathrm{mmol} / \mathrm{L})$ e aos cordeiros únicos (2,17mmol/L). Segundo Stafford et al. (2007), não há diferença no lactato de acordo com a ordem de nascimento dos cordeiros (primeiro, segundo ou terceiro cordeiro) ou o peso (cordeiros pequenos, médios ou grandes).
A maior concentração de lactato em cordeiros gêmeos oriundos de parto eutócico provavelmente se correlaciona à menor pressão parcial de $\mathrm{O}_{2}, \mathrm{pH}, \mathrm{HCO}_{3}{ }^{-}$e excesso de base (BE) e maior concentração do $\mathrm{CO}_{2}$ total ao nascimento, representando um maior risco para o desenvolvimento de asfixia em relação aos cordeiros únicos, conforme descrito por Banchero e Dutra (2011). A área de superfície dos cotilédones placentários declina com o maior número de fetos por ninhada, promovendo uma redução da eficiência cotiledonária. No terço final da gestação, o desenvolvimento de acidemia metabólica junto à insuficiência placentária é observado nos partos gemelares, aumentando o risco de asfixia em até 15,6 vezes a mais em cordeiros gêmeos (Sparks et al., 1983; Dutra e Banchero, 2011).

Não houve diferença estatística entre o lactato das ovelhas e o dos cordeiros, sendo os resultados do presente estudo semelhantes aos descritos por Sparks et al. (1982) e Comline e Silver (1972), no que se refere à elevação do lactato materno e do neonatal, no entanto tais resultados diferiram quanto à magnitude da elevação. Segundo os autores, o aumento de lactato no plasma materno é menor quando 
comparado ao dos cordeiros recém-nascidos, representando apenas um terço deste.

Houve correlação positiva entre a concentração de lactato materno e dos cordeiros após o parto eutócico (Fig. 1). Os resultados do presente estudo diferiram dos propostos por Alexander, Moinhos e Scott (1968), os quais descreveram que as alterações do lactato na ovelha e no feto no momento do parto refletem a diferença entre o efeito da descarga adrenérgica no adulto, no feto e no neonato.

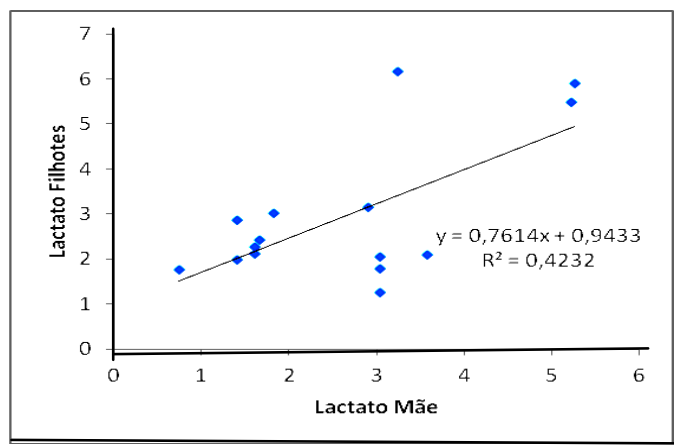

Figura 1. Correlação entre a concentração de lactato materno e dos cordeiros nascidos de parto eutócico.

Durante o parto, as alterações hemodinâmicas e metabólicas maternas resultam em grande parte da estimulação hormonal (cortisol e catecolaminas) promovida pela dor, ansiedade e contrações uterinas (Song et al., 2004). A elevação da concentração de lactato na fêmea imediatamente após o parto representa, portanto, um reflexo da descarga hormonal adrenérgica que ocorreu previamente. Durante o parto, a descarga adrenérgica materna promove vasoconstrição periférica, e a produção de lactato também se eleva no feto em decorrência do metabolismo anaeróbico promovido pela hipóxia (Alexander, Moinhos e Scott, 1968; Miley, 1997). Isto poderia explicar a correlação entre o lactato materno e o neonatal.

\section{CONCLUSÃO}

Os resultados do presente estudo demonstraram que a concentração sérica de lactato em cordeiros neonatos oriundos de eutocia encontra-se elevada no pós-parto imediato, portanto sua utilização como fator prognóstico, capaz de refletir indiretamente os níveis de perfusão tecidual em neonatos, deve ser feita com cautela para caracterização do estado de hipóxia intraparto, uma vez que os valores obtidos em tal momento podem simplesmente refletir o padrão fisiológico para esta fase de transição fetal-neonatal na espécie ovina. A hiperlactatemia deve ser interpretada junto a outros índices, como o de viabilidade neonatal (escore de Apgar), bem como a hemogasometria.

\section{AGRADECIMENTOS}

Ao Setor de Ovinocultura da Fazenda Experimental "Marcelo Mesquita Serva" da Universidade de Marília (Unimar), pelo fornecimento dos animais deste estudo.

\section{REFERÊNCIAS}

ALEXANDER, G.; MILLS, S.C.; SCOTT, T.W. Changes in plasma glucose, lactate and free fatty acids in lambs during summit metabolism and treatment with catecholamines. J. Physiol., v.198, p.277-289, 1968.

BELETTINI, S.T; ALBERTON, L.R; SILVA, R.P.B. et al. Avaliação dos níveis séricos de lactato em cães submetidos a anestesia dissociativa. Arq. Cienc. Vet. Zool., v.11, p.8795, 2008 .

BLEUL, U.; SACHWATANG, S.; KANHN, W. Blood gas analysis of bovine fetal capillary blood during stage II of labor. Theriogenology, v.69, p.245-251, 2008.

CLOETE, S.W.P.; SCHOLTZ, A.J. Lamb survival in relation to lambing and neonatal behaviour in medium wool Merino lines divergently selected for multiple rearing ability. Aust. J. Exp. Agric., v.38, p.801-811, 1998.

COMLINE, R.S.; SILVER, M. The composition of foetal and maternal blood during parturition in the ewe. J. Physiol., v.222, p.233-256, 1972.

DUTRA, F.; QUINTANS, G.; BANCHERO, G. Lesions in the central nervous system associated with perinatal lamb mortality. Aust. Vet. J., v.85, p.405-413, 2007.

DUTRA, F.; BANCHERO, G. Polwarth and texel ewe parturition duration and its association with lamb birth asphyxia. J. Anim. Sci., v.89, p.3069-3078, 2011.

DWYER, C.M. The welfare of the neonatal lamb. Small Rum. Res., v.76, p.31-41, 2008. 
EVERETT-HINCKS， J.M.; DODDS， K.G.; KERSLAKE, J.I. Parturition duration and birthing difficulty in twin and triplet lambs. Proc. N. Z. Soc. Anim. Prod.,v.67, p.55-60, 2007.

FLORIANO, B.P.; OLIVEIRA, G.C.V.; VIVAN, M.C.R. et al. Lactato sanguíneo na avaliação dos efeitos da peridural torácica em cães anestesiados pelo isofluorano. Cienc. Rural, v.40, p.574-579, 2010.

GROPPETTI, D.; PECILE, A.; DEL CARRO, A.P. et al. Evaluation of newborn canine viability by means of umbilical vein lactate measurement, apgar score and uterine tocodynamometry. Theriogenology, v.20, p.1187-1196, 2010.

HERPIN, P.; LE DIVIDICH, J.; HULIN, J.C.; FILLAUT, M. et al. Effects of the level of asphyxia during delivery on viability at birth and early postnatal vitality of newborn pigs. J. Anim. Sci., v.74, p.2067-2075, 1996.

KANEKO, J.J. Lipids and ketones. In: KANEKO, J.J. Clinical biochemistry of domestic animals. 5.ed. San Diego: Academic, 1997. p.83115.

KOLISKI, A.; CAT, I.; GIRALDI, D.J.; CAT, M.L. Blood lactate concentration as a prognostic marker in critically ill children. Rev.chil.pediatr., v.78, p.321-329, 2007.

LOW, J.A. Metabolic acidosis and fetal reserve. Baillieres Clin. Obstet. Gynaecol., v.10, p.211224, 1996.

MAGDESIAN, GK. Blood lactate levels in neonatal foals: normal values and temporal effects in the post-partum period. J. Vet. Emerg. Crit. Care., v.13, p.174, 2003

MILEY, J.R. Ovine fetal metabolism during norepinephrine infusion. Am. J. Physiol. Endocrinol. Metab., v.273, p.E336-E347, 1997.

PEIRÓ, J.R.; BORGES, A.S.; GONÇALVES, R.C.; MENDES, L.C.N. Evaluation of a portable clinical analyzer for the determination of blood gas partial pressures, electrolyte concentrations, and hematocrit in venous blood samples collected from cattle, horses, and sheep. Am. J. Vet. Res., v.71, p.515-521, 2010.
RANDALL, G.C. The relationship of arterial blood $\mathrm{pH}$ and $\mathrm{pCO} 2$ to the viability of the newborn piglet. Can. J. Comp. Med., v.35, p.141-146, 1971.

ROOK, J.S.; SCHOLMAN, G.; WINGPROCTOR, S.; SHEA, M.E. Diagnosis and control of neonatal losses in sheep. Vet. Clin. North Am.: Food Anim. Pract., v.6, p.531-562, 1990.

SAUGSTAD, O.D. Is lactate a reliable indicator of tissue hypoxia in the neonatal period? Acta Paediatr., v.91, p.17-19, 2002.

SONG, J.; ZHANG, S.; QIAO, Y.; LUO, Z. et al. Predicting pregnancy-induced hypertension with dynamic hemodynamics. Eur. Obs. Gynecol. Repro. Biol., v.117, p.162-168, 2004.

SOUZA, M.H.L.; ELIAS, D.O. Valor prognóstico da acidose láctica durante a perfusão. Rer. Latinoamer. Tecnol. Extra., v.13, p.14-17, 2006.

SPARKS, J.W.; HAY, W.W. Jr.; BONDS, D.; MESCHIA, G. Simultaneous measurements of lactate turnover rate and umbilical lactate uptake in the fetal lamb. J. Clin. Invest., v.70, p.179192, 1982.

SPARKS, J.W.; HAY, W.W. JR.; MESCHIA, G.; BATTAGLIA, F.C. Partition of maternal nutrients to the placenta and fetus in the sheep. Europ. J. Obstet. Gynec. Reprod. Biol., v.14, p.331-340, 1983.

STAFFORD, K.J.; KENYON, P.R.; MORRIS, S.T.; WEST, D.M. The physical state and metabolic status of lambs of different birth rank soon after birth. Liv. Sci., v.111, p.10-15, 2007.

THORNGREN-JERNECK, K.; LEY, D.; HELLSTRÖM-WESTAS, L. et al. Reduced postnatal cerebral glucose metabolism measured by PET after asphyxia in near term fetal lambs. J. Neuro. Res., v.66, p.884-850, 2001.

VAALA, W.E. Peripartum asphyxia syndrome in foals. Proc. Am. Assoc. Equine Pract., v.45, p.247-253, 1999.

VOLPE, J.J. Hypoxic-ischemic encephalopathy. In: Volpe, J.J. (Ed). Neurology of the Newborn. Philadelphia: W. B. Saunders Company, 2001. p.217-394. 